\title{
Arginine vasopressin neuronal loss results from autophagy-associated cell death in a mouse model for familial neurohypophysial diabetes insipidus
}

\author{
D Hagiwara ${ }^{1}$, H Arima ${ }^{*, 1}$, Y Morishita ${ }^{1}$, L Wenjun ${ }^{1}$, Y Azuma ${ }^{1}$, Y Ito ${ }^{1}$, H Suga ${ }^{1}$, M Goto ${ }^{1}$, R Banno ${ }^{1}$, Y Sugimura ${ }^{1}$, A Shiota ${ }^{2}$, \\ N Asai ${ }^{3}$, M Takahashi ${ }^{3}$ and Y Oiso ${ }^{1}$
}

Familial neurohypophysial diabetes insipidus (FNDI) characterized by progressive polyuria is mostly caused by mutations in the gene encoding neurophysin II (NPII), which is the carrier protein of the antidiuretic hormone, arginine vasopressin (AVP). Although accumulation of mutant NPII in the endoplasmic reticulum (ER) could be toxic for AVP neurons, the precise mechanisms of cell death of AVP neurons, reported in autopsy studies, remain unclear. Here, we subjected FNDI model mice to intermittent water deprivation (WD) in order to promote the phenotypes. Electron microscopic analyses demonstrated that, while aggregates are confined to a certain compartment of the ER in the AVP neurons of FNDI mice with water access ad libitum, they were scattered throughout the dilated ER lumen in the FNDI mice subjected to WD for 4 weeks. It is also demonstrated that phagophores, the autophagosome precursors, emerged in the vicinity of aggregates and engulfed the ER containing scattered aggregates. Immunohistochemical analyses revealed that expression of p62, an adapter protein between ubiquitin and autophagosome, was elicited on autophagosomal membranes in the AVP neurons, suggesting selective autophagy induction at this time point. Treatment of hypothalamic explants of green fluorescent protein (GFP)-microtubule-associated protein 1 light chain 3 (LC3) transgenic mice with an ER stressor thapsigargin increased the number of GFP-LC3 puncta, suggesting that ER stress could induce autophagosome formation in the hypothalamus of wild-type mice as well. The cytoplasm of AVP neurons in FNDI mice was occupied with vacuoles in the mice subjected to WD for 12 weeks, when 30-40\% of AVP neurons are lost. Our data thus demonstrated that autophagy was induced in the AVP neurons subjected to ER stress in FNDI mice. Although autophagy should primarily be protective for neurons, it is suggested that the organelles including ER were lost over time through autophagy, leading to autophagy-associated cell death of AVP neurons.

Cell Death and Disease (2014) 5, e1148; doi:10.1038/cddis.2014.124; published online 27 March 2014

Subject Category: Experimental Medicine

Arginine vasopressin (AVP), an antidiuretic hormone, is synthesized in the magnocellular neurons in the supraoptic nucleus (SON) and paraventricular nucleus of the hypothalamus. ${ }^{1}$ The AVP gene encodes the signal peptide, AVP, neurophysin II (NPII), which is the carrier protein of AVP, and glycoprotein. The AVP precursor (preproAVP) is converted to proAVP by the removal of the signal peptide within the endoplasmic reticulum (ER), followed by proAVP folding. AVP, NPII and glycoprotein are cleaved from proAVP in the vesicle and are transported to the posterior pituitary, from where AVP is released into the systemic circulation. ${ }^{2}$

Familial neurohypophysial diabetes insipidus (FNDI) is an autosomal dominant disorder caused by mutations in the AVP gene locus, mostly in the NPII-coding region, and patients with FNDI manifest progressive polyuria several months or years after birth. ${ }^{3}$ There are several lines of evidence to suggest that the mutant AVP precursors are trapped in the ER, ${ }^{4-6}$ and our previous studies demonstrated that inclusion bodies were formed in the lumen of ER in a FNDI mouse model. ${ }^{7,8}$ The accumulation of aggregates in the ER would finally lead to cellular loss of AVP neurons, as previous autopsy studies reported AVP neuronal loss in the hypothalamus of patients with FNDI. ${ }^{9-11}$ However, the mechanisms by which AVP neurons are lost remain to be clarified.

Although ER stress, which could be caused by the accumulation of misfolded proteins in the $E R$, has been implicated in many diseases, ${ }^{12}$ there are only limited numbers of animal models for diseases, such as $\alpha_{1}$-antitrypsin deficiency, ${ }^{13}$ familial encephalopathy with neuroserpin inclusion body, ${ }^{14}$ neonatal diabetes mellitus ${ }^{15}$ and FNDI, in which aggregate accumulation in the ER lumen is visible. These animal models would therefore be useful to study how the ER

\footnotetext{
${ }^{1}$ Department of Endocrinology and Diabetes, Nagoya University Graduate School of Medicine, Nagoya, Japan; ${ }^{2}$ PhoenixBio Co. Ltd, Utsunomiya, Japan and ${ }^{3}$ Department of Pathology, Nagoya University Graduate School of Medicine, Nagoya, Japan

*Corresponding author: H Arima, Department of Endocrinology and Diabetes, Nagoya University Graduate School of Medicine, 65 Tsurumai-cho, Showa-ku, Nagoya 466-8550, Japan. Tel: +81 52744 2142; Fax: +81 52744 2206; E-mail: arima105@med.nagoya-u.ac.jp

Keywords: autophagy; autophagy-associated cell death; ER stress; ER-associated compartment; familial neurohypophysial diabetes insipidus

Abbreviations: FNDI, familial neurohypophysial diabetes insipidus; AVP, arginine vasopressin; NPII, neurophysin II; SON, supraoptic nucleus; WD, water deprivation; ER, endoplasmic reticulum; ERAC, endoplasmic reticulum-associated compartment; BiP, immunoglobulin heavy chain binding protein; UPS, ubiquitin-proteasome system; GFP, green fluorescent protein; LC3, microtubule-associated protein 1 light chain 3; hnRNA, heteronuclear RNA; TUNEL, terminal deoxynucleotidyl transferasemediated deoxyuridine triphosphate nick end labeling

Received 21.12.13; revised 26.2.14; accepted 27.2.14; Edited by A Verkhratsky
} 
stress is related to cellular dysfunction and finally causes cell death. However, life spans of animals could be a barrier to study the cell death in vivo. This is also the case with the FNDI mouse model: while the FNDI mice recapitulated the phenotype of patients with FNDI by showing progressive polyuria because of deficiency of AVP, the AVP neuronal loss is minimal, if any, even at the age of 12 months. ${ }^{7}$ In order to explore the mechanisms of cell death in vivo, it would be also important to synchronize neurons toward cell death: to analyze the mechanisms of cell death would be difficult if cells are lost gradually over time.

In this study, we subjected the FNDI mice to intermittent water deprivation (WD), in order to promote the accumulation of mutant AVP precursors and possibly accelerate the phenotypes, and examined the process and mechanisms of AVP neuronal loss under ER stress in FNDI mice.

\section{Results}

The phenotype of FNDI mice is accelerated under WD. There were no significant differences in urine volumes between ad libitum and WD groups in wild-type (WT) mice (Figure 1a). On the other hand, urine volumes were significantly increased in the WD group compared with the ad libitum group in FNDI mice at 5-12 weeks after starting WD (Figure 1a). Urine AVP in the WD group was significantly increased compared with the ad libitum group in WT mice at 12-week (Figure 1b), a possible response to chronic dehydration. In contrast, urine AVP in the WD group was significantly decreased than that in the ad libitum group in FNDI mice at 12-week (Figure 1c). Thus, WD accelerated the phenotype of polyuria because of AVP deficiency in the FNDI mice.

Aggregates confined to a certain compartment of the ER in AVP neurons of FNDI mice are scattered throughout the dilated ER under WD. Although round inclusion bodies were observed in AVP neurons of the SON in both ad libitum and WD groups in 3-month-old FNDI mice, the sizes were smaller in the mice subjected to WD for 4 weeks (Figure 2a). The number of inclusion bodies with a diameter $>4.5 \mu \mathrm{m}$, the mean size of inclusion bodies in the ad libitum group, was also decreased in the FNDI mice subjected to WD (Figure 2b), suggesting that the inclusion body formation is hampered under WD. Electron microscopic analyses showed massive and round-shaped electron-dense aggregates in the AVP neurons in FNDI mice with water access ad libitum (Figure 3a), as reported previously, ${ }^{7}$ while the lumen of the ER adjacent to the massive aggregates was preserved intact (Figure $3 b$ ). There were also irregular-shaped aggregates confined to some compartments of the ER (Figure 3c), which communicate with intact ER lumen (Figure 3d, arrowheads). These observations suggest that aggregates are confined to a certain compartment of the ER in the AVP neurons of FNDI mice with water access ad libitum. In contrast, smaller-sized aggregates were scattered throughout the dilated ER lumen in AVP neurons in the FNDI mice subjected to WD for 4 weeks (Figures $3 e$ and $\mathrm{f}$ ). It is also demonstrated that phagophores, the autophagosome precursors, emerged in the vicinity of aggregates and engulfed the ER containing scattered aggregates (Figures $3 g$ and $h$ ).
Confined aggregate formation alleviates ER stress in AVP neurons of FNDI mice. Despite the presence of massive aggregates formed in a sub-compartment of the ER lumen of AVP neurons in FNDI mice, there was no significant difference in the expression levels of immunoglobulin heavy chain binding protein (BiP) mRNA between FNDI and WT mice with water access ad libitum (Supplementary Figure S1a). On the other hand, the expression levels of BiP mRNA were significantly increased in FNDI mice compared
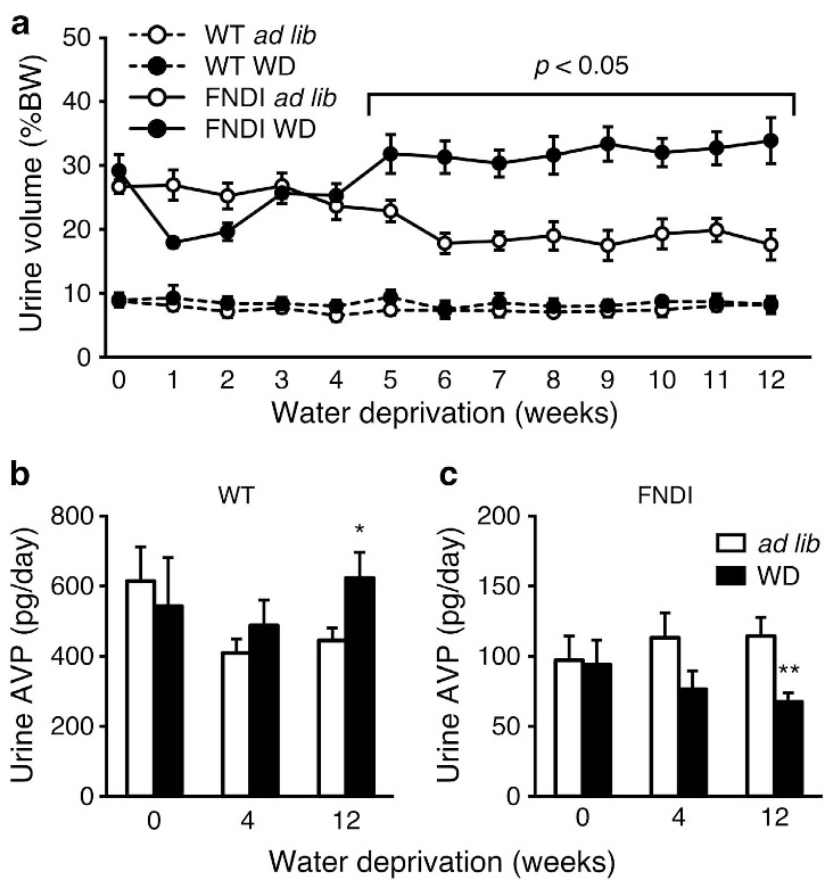

Figure 1 Changes in urine volume and AVP. (a) Urine volumes in WT (dashed line) and FNDI mice (solid line) with water access ad libitum (ad lib, open circles) or subjected to WD (closed circles). (b and c) Urine AVP in ad lib and WD groups in WT (b) and FNDI mice (c). Results are expressed as means \pm S.E. ${ }^{*} P<0.05$, ${ }^{\star \star} P<0.01$ compared with ad lib group at each corresponding time point. $n=5-6$ animals per group. \% BW, percent body weight
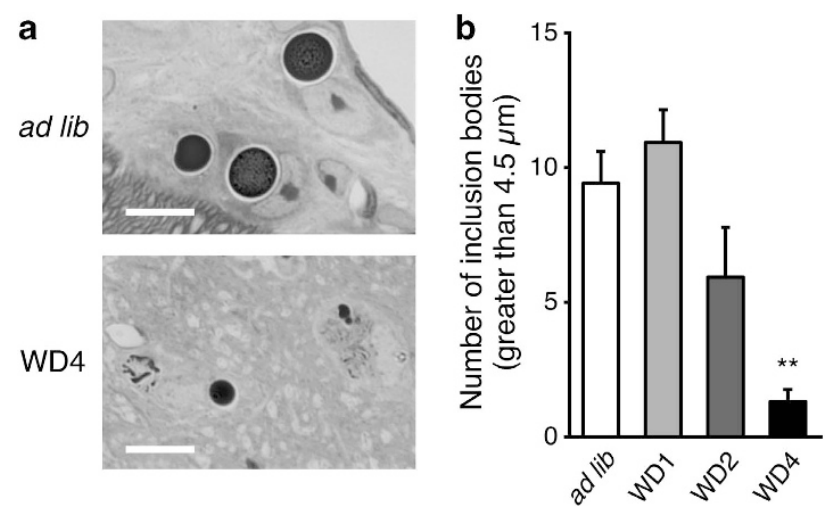

Figure 2 Effects of WD on inclusion body formation in AVP neurons of FNDI mice. (a) Toluidine blue staining of the SON in 3-month-old FNDI mice with water access ad libitum (ad lib) or WD for 4 weeks (WD4). (b) The number of inclusion bodies with a diameter $>4.5 \mu \mathrm{m}$ in the SON. The data of 3-month-old FNDI mice with water access ad libitum (ad lib) and of those subjected to WD for 1, 2, and 4 weeks (WD1, 2, 4) are shown. Results are expressed as means \pm S.E. ${ }^{* *} P<0.01$ compared with ad lib group. $n=5-7$ animals per group. Scale bars, $10 \mu \mathrm{m}$ 

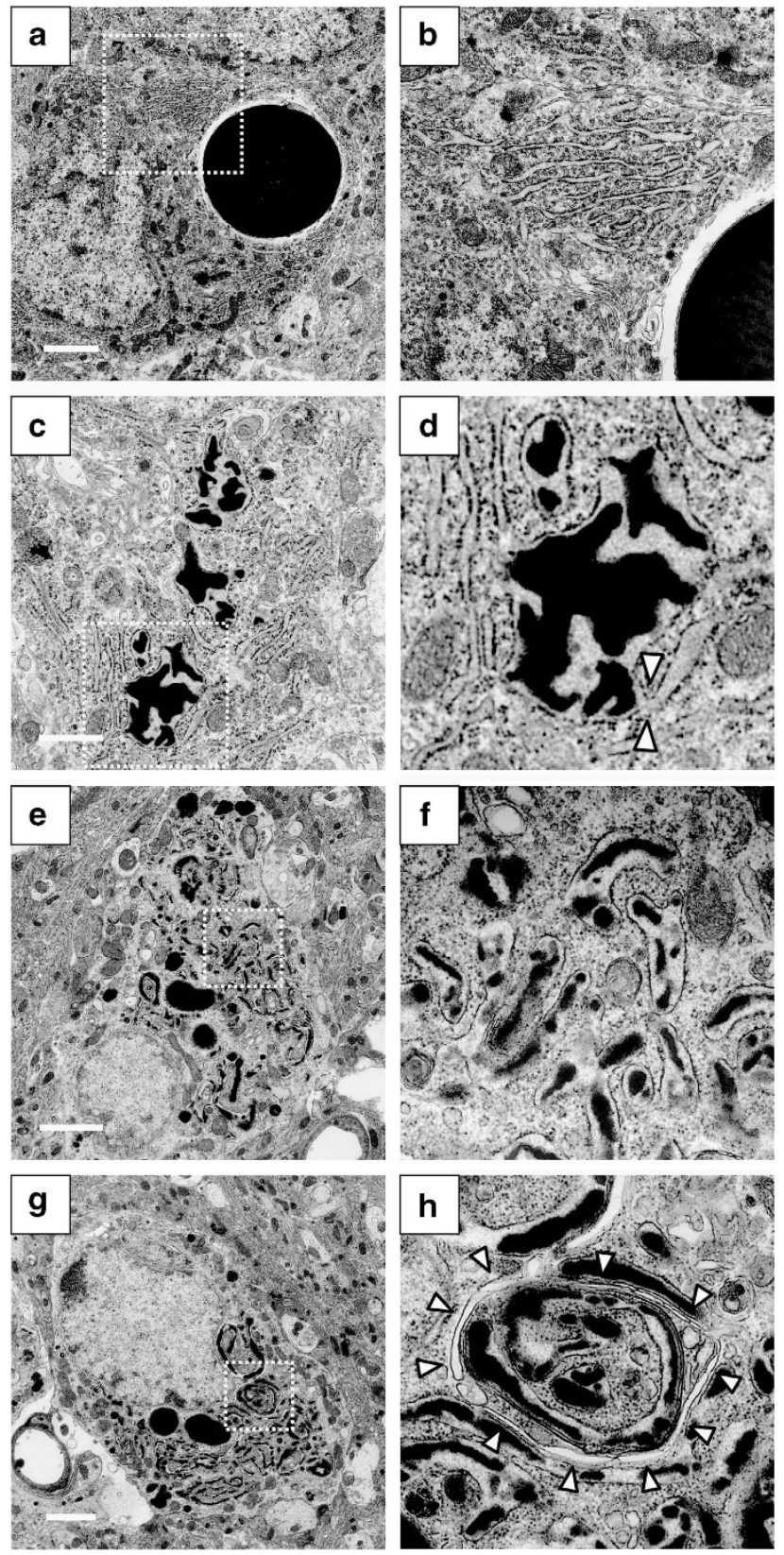

Figure 3 Electron microscopic analysis in AVP neurons of 3-month-old FNDI mice. AVP neurons of the SON in 3-month-old FNDI mice with water access ad libitum (a-d) and of those subjected to WD for 4 weeks $(\mathbf{e}-\mathbf{h})$. Higher magnification images of boxed areas in (a, $\mathbf{c}, \mathbf{e}$ and $\mathbf{g})$ are shown in ( $\mathbf{b}, \mathbf{d}, \mathbf{f}$ and $\mathbf{h})$, respectively. The arrowheads in (d) indicate irregular-shaped aggregates confined to some compartments of the ER, which seem to communicate with intact ER lumen, and those in $(\mathbf{h})$ indicate phagophores engulfing the $E R$, which contains scattered aggregates. Scale bars, $2 \mu \mathrm{m}$ (a, e and $\mathbf{g}), 1 \mu \mathrm{m}$ (c)

with those in WT mice subjected to WD for 4 weeks (Supplementary Figures S1a and b).

Autophagy is induced while ubiquitinated proteins are not accumulated in AVP neurons of FNDI mice under WD. Although virtually no green fluorescent protein (GFP) signals were detected in AVP neurons of the SON in FNDI/ GFP-microtubule-associated protein 1 light chain 3 (LC3) a
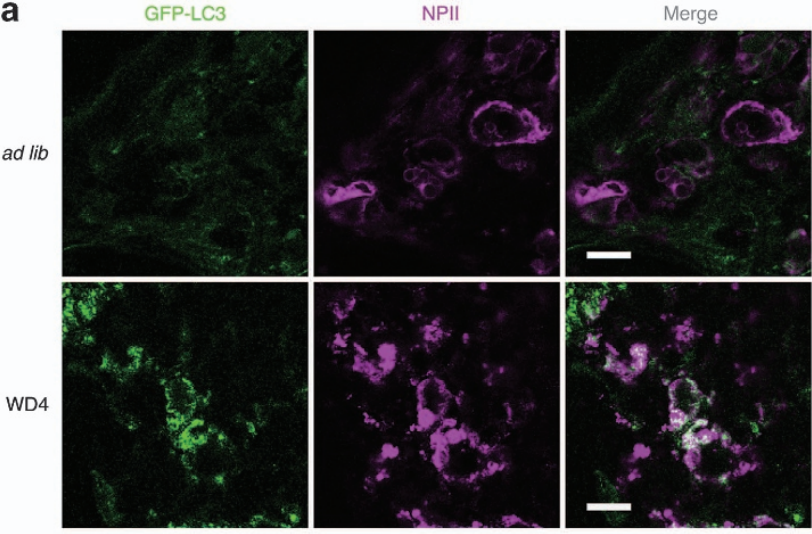

b
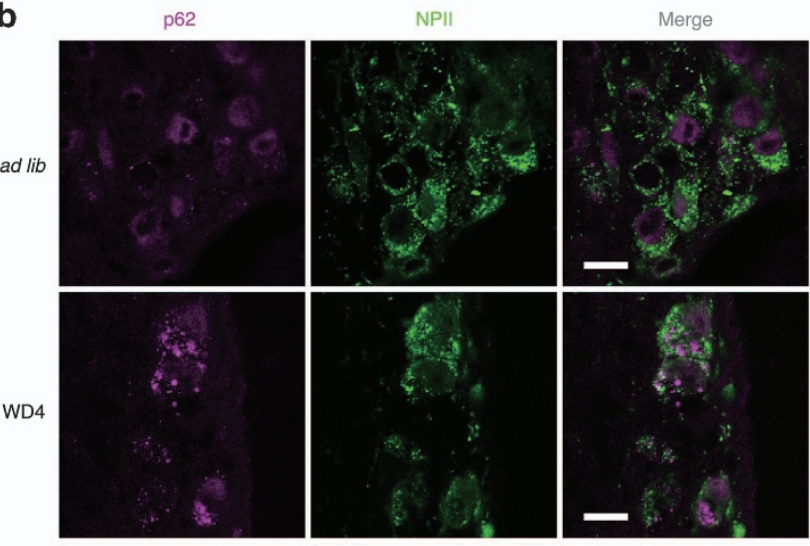

C

p62
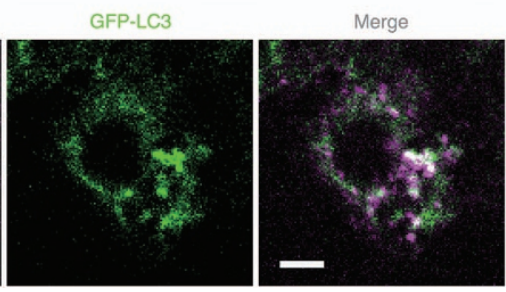

Figure 4 Autophagosome formation and p62 expression in AVP neurons of FNDI/GFP-LC3 mice. (a) Immunofluorescence staining for GFP (green) and mutant NPII (magenta) in the SON of 3-month-old FNDI/GFP-LC3 mice with water access ad libitum (ad lib) and of those subjected to WD for 4 weeks (WD4). (b) Immunofluorescence staining for p62 (magenta) and NPII (green) in the SON of 3-month-old FNDI mice in ad lib and WD4 groups. (c) Immunofluorescence staining for p62 (magenta) and GFP-LC3 (green) in the SON of 3-month-old FNDI/GFP-LC3 mice in WD4 group. Scale bars, $10 \mu \mathrm{m}$ (a and b), $5 \mu \mathrm{m}$ (c)

mice with water access ad libitum, GFP signals were elicited in the AVP neurons in the mice subjected to WD for 4 weeks (Figure 4a). p62/sequestosome-1 is an adapter protein that binds to LC3 and ubiquitin, and is therefore thought to be a receptor for ubiquitinated cargos to deliver to the autophagosomes in selective autophagy. ${ }^{16}$ Immunofluorescence images revealed that p62 expression was elicited in AVP neurons of the SON in FNDI mice subjected to WD for 4 weeks (Figure 4b). Furthermore, the majority of enhanced fluorescence signals of $p 62$ were colocalized with GFP signals in FNDI/GFP-LC3 mice subjected to WD for 4 weeks (Figure 4c). On the other hand, GFP signals were barely detected in AVP neurons of FNDI/Ub ${ }^{\mathrm{G} 76 \mathrm{~V}}$-GFP mice with water access ad libitum or subjected to WD (Supplementary 

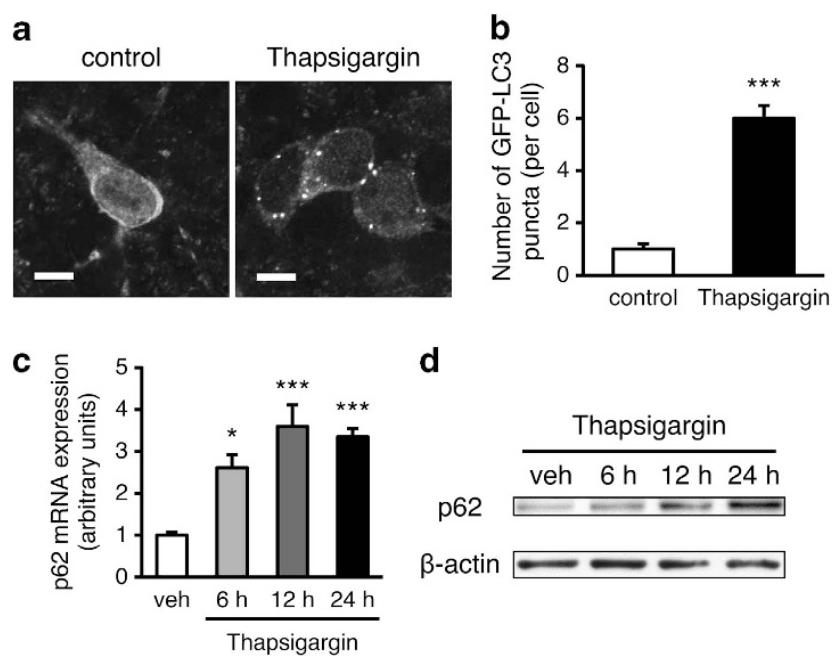

d

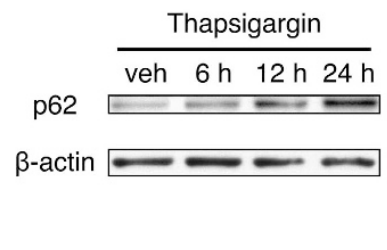

Figure 5 Autophagosome formation and p62 expression under ER stress in mouse hypothalamic slice explants. (a) Immunofluorescence staining for GFP in hypothalamic slice explants of GFP-LC3 transgenic mice cultured with vehicle (control) and $1 \mu \mathrm{M}$ thapsigargin for $24 \mathrm{~h}$. (b) The number of GFP-LC3 puncta per cell. GFP-LC3 puncta in 60-70 cells were counted in each group in a series of equally acquired images. (c) Quantitative real-time RT-PCR analysis for p62 mRNA in hypothalamic slice explants of WT mice cultured with vehicle (veh) and $1 \mu \mathrm{M}$ thapsigargin for 6, 12 and $24 \mathrm{~h}$. (d) Immunoblot analysis of protein lysates from hypothalamic slice explants of WT mice immunolabeled for p62. Explants were incubated with vehicle (veh) and $1 \mu \mathrm{M}$ thapsigargin for 6,12 and $24 \mathrm{~h}$. Results are expressed as means \pm S.E. ${ }^{*} P<0.05,{ }^{* * *} P<0.001$ compared with vehicle-treated explants. Scale bars, $10 \mu \mathrm{m}$

Figure S2a), while they were elicited in hypothalamic slice explants of $\mathrm{Ub}^{\mathrm{G} 76 \mathrm{~V}_{-}}$-GFP transgenic mice treated with a proteasome inhibitor MG-132 (Supplementary Figure S2b). These data suggest that ubiquitinated proteins were not accumulated in the AVP neurons in FNDI mice.

ER stress induces autophagosome formation in mouse hypothalamic slice explants. The findings in electron microscopic analyses and FNDI/GFP-LC3 mice suggest that ER stress induced autophagy in the AVP neurons of the SON in FNDI mice subjected to WD. To determine whether or not this is a phenomenon only seen in the AVP neurons in FNDI mice, we treated hypothalamic explants of GFP-LC3 transgenic mice with an ER stressor thapsigargin. Immunofluorescence staining revealed that the number of GFP-LC3 puncta was increased in explants exposed to thapsigargin (Figures 5a and b). In addition, p62 expression was increased at both mRNA and protein levels in the hypothalamic explants treated with thapsigargin (Figures $5 c$ and d). These findings suggest that ER stress could lead to autophagosome formation and increase p62 expression in the hypothalamus of WT mice as well.

AVP neuronal loss is promoted under WD in FNDI mice. The number of AVP neurons in the SON of 5-monthold FNDI mice subjected to WD for 12 weeks was decreased $30-40 \%$ compared with that of the FNDI mice with water access ad libitum (Figures 6a-f). On the other hand, the AVP heteronuclear RNA (hnRNA) grains per cell in 5-month-old FNDI mice subjected to WD for 12 weeks were significantly increased compared with those in the FNDI mice with water access ad libitum (Figures $6 \mathrm{~g}-\mathrm{i}$ ), suggesting that the transcriptional activities in the residual AVP neurons were preserved even at this stage when AVP neurons are dying. Terminal deoxynucleotidyl transferase-mediated deoxyuridine triphosphate nick end labeling (TUNEL) assay in the SON in 5-month-old FNDI mice subjected to WD for 12 weeks was negative (data not shown).

AVP neuronal loss is associated with autophagy in FNDI mice. Electron microscopic analyses in 5-month-old FNDI mice with water access ad libitum showed massive and round-shaped aggregates, as seen in 3-month-old FNDI mice with water access ad libitum (Figure 7a). However, the lumen of ER was dilated throughout the cells at this age (Figure 7b). There were also scattered aggregates in dilated ER lumen (Figures $7 \mathrm{c}$ and $\mathrm{d}$ ), a finding similar to that seen in the AVP neurons of 3-month-old FNDI mice subjected to WD. In FNDI mice subjected to WD for 12 weeks, there were vacuoles in the cytoplasm while the nuclear structure was relatively preserved (Figures $7 e$ and f). In some cells, huge vacuoles with aggregates inside were observed (Figures $7 \mathrm{~g}$ and $\mathrm{h}$ ).

\section{Discussion}

In this study, we promoted the phenotypes of FNDI mice by subjecting them to intermittent WD, and succeeded in making an animal model to analyze how ER stress leads to cell death in mice.

Electron microscopic analyses in this study clearly demonstrated that, while there were massive aggregates in a subcompartment of ER lumen of AVP neurons in the FNDI mice with water access ad libitum, there was intact ER lumen adjacent to the aggregates. Certain misfolded secretory or transmembrane proteins (destined to be degraded) are reportedly segregated into specialized sub-compartments of ER in yeast ${ }^{17}$ or cell lines. ${ }^{18-21}$ Such structures have been called Russell body, ${ }^{18}$ quality control compartment, ${ }^{19}$ ER-derived protective organelle ${ }^{21}$ or ER-associated compartment $^{17}$ (hereafter, referred to as ERAC). It is shown that ERAC formation does not perturb processing of normal secretory and transmembrane proteins nor induce unfolded protein response in yeast expressing a mutant Ste6p, a multispanning membrane protein, ${ }^{17}$ or in hepatoma cell lines expressing a mutant $\alpha_{1}$-antitrypsin. ${ }^{20}$ Consistent with these previous studies, our data demonstrated that, in spite of the presence of massive aggregates in the ER of AVP neurons in FNDI mice, the expression levels of BiP mRNA were similar between FNDI and WT mice with water access ad libitum, suggesting that ERAC formation alleviates ER stress possibly caused by the accumulation of mutant proteins in AVP neurons in FNDI mice. Our data also demonstrated that aggregates were scattered throughout the dilated ER lumen in AVP neurons in the 3-month-old FNDI mice subjected to WD. As similar findings were found in 5-month-old (in this study) and 12-month-old FNDI mice with water access ad libitum, ${ }^{7}$ it is suggested that such phenomena could occur over time even in FNDI mice with water access ad libitum. Failure to confine aggregates to a sub-compartment of ER lumen 
a

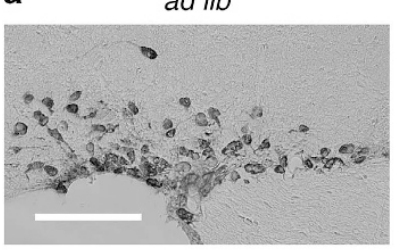

d

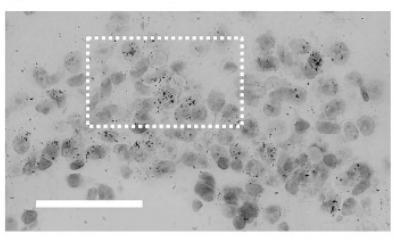

g

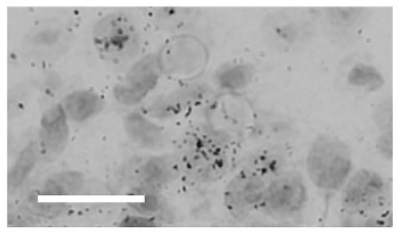

b

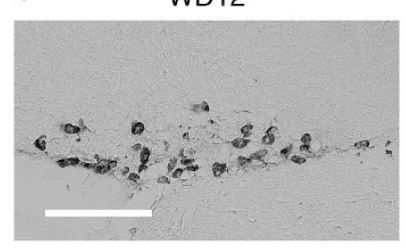

e

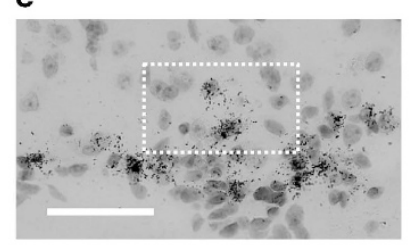

h

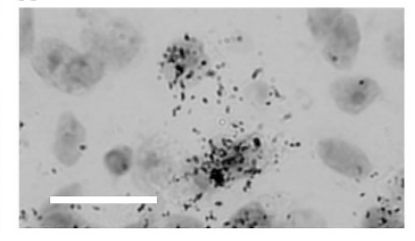

C

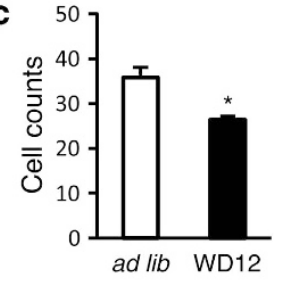

f
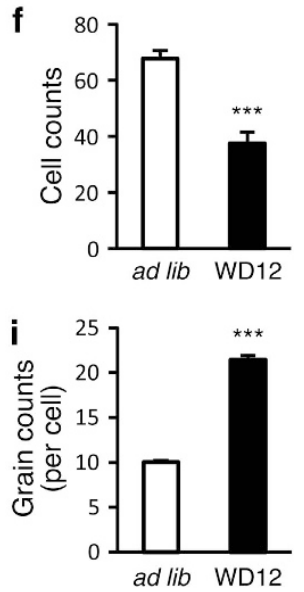

Figure 6 AVP neuronal loss in FNDI mice subjected to WD. (a and $\mathbf{b}$ ) Immunohistochemistry for NPII in the SON of 5-month-old FNDI mice with water access ad libitum (ad lib) and of those subjected to WD for 12 weeks (WD12). (c) The number of NPII-expressing cells in the SON counted with immunohistochemistry. (d and e) In situ hybridization for AVP hnRNA in the SON of 5-month-old FNDI mice in ad lib and WD12 groups. (f) The number of AVP hnRNA-expressing cells in the SON counted with in situ hybridization. ( $\mathbf{g}$ and $\mathbf{h}$ ) Higher magnification images of boxed areas in ( $\mathbf{d}$ and $\mathbf{e}$ ), respectively. (i) The numbers of AVP hnRNA grains per cell are shown. Results are expressed as means \pm S.E. ${ }^{*} P<0.05,{ }^{* \star *} P<0.001$ compared with ad lib group. $n=5-8$ animals per group. Scale bars, $100 \mu \mathrm{m}(\mathbf{a}$ and $\mathbf{b}), 50 \mu \mathrm{m}(\mathbf{d}$ and $\mathbf{e}), 20 \mu \mathrm{m}(\mathbf{g}$ and $\mathbf{~})$

resulted in increases in ER stress as demonstrated by increases in BiP mRNA expression in FNDI mice compared with WT mice subjected to WD.

Possible involvement of autophagy in the pathogenesis of FNDI has been suggested in previous studies both in vitro ${ }^{22-24}$ and in vivo. ${ }^{25,26}$ In this study, we clearly demonstrated that autophagy is induced when ERAC failed to form, thereby resulting in increased ER stress. The hypothesis that ER stress per se triggered autophagy in AVP neurons in vivo was supported by the findings that an ER stressor induced autophagy in hypothalamic organotypic cultures in GFP-LC3 transgenic and WT mice. It is also of note that the targets of autophagy include the ER of AVP neurons in FNDI mice. It is reported that ER stress induced autophagy in yeast ${ }^{27}$ and mammalian cell lines. ${ }^{28}$ Furthermore, autophagy targeting for $E R$, called ER-phagy, is also shown to be induced in yeast by ER stressors ${ }^{29}$ and in mammalian cell lines expressing mutant $\alpha_{1}$-antitrypsin. ${ }^{30,31}$ However, this is the first report showing ER-phagy was induced in the neurons in vivo, to the best of our knowledge.

In general, aberrant or misfolded proteins in the ER are degraded by the ER-associated degradation, in which substrates are retrotranslocated to the cytoplasm and degraded by the ubiquitin-proteasome system (UPS). ${ }^{32}$ On the other hand, it is also suggested that ubiquitinated proteins are degraded via autophagic machinery as well, which is called selective autophagy. ${ }^{33}$ Crosstalk between UPS and autophagy is provided by autophagic adapter proteins such as p62, which bind ubiquitin and autophagosome membrane LC3. It is known that p62 accumulation could be induced by inhibition of proteasome ${ }^{34,35}$ or autophagy. ${ }^{36}$ However, the experiments using FNDI/Ub ${ }^{G 76 V_{-G F}}$ mice in this study demonstrated that the proteasomal degradation is intact in the AVP neurons in FNDI mice. Furthermore, p62 was upregulated at mRNA levels by an ER stressor in hypothalamic explants of WT mice, and autophagy in the AVP neurons seemingly continued in the FNDI mice subjected to WD for 12 weeks, as discussed below. Thus, it is suggested that p62 expression in the FNDI mice subjected to WD for 4 weeks was transcriptionally upregulated by ER stress, as has been reported in a previous study. ${ }^{37}$

The intermittent WD for 12 weeks resulted in 30-40\% AVP neuronal loss in FNDI mice in this study. The types of cell death are classified into three groups based on the morphology: apoptosis, necrosis and autophagic cell death. ${ }^{38,39}$ TUNEL assay in the SON was negative in these mice and electron microscopic analyses revealed that the nuclear structure was relatively preserved. Increases in AVP hnRNA expression, an indicator of gene transcription, in FNDI mice subjected to WD for 12 weeks also suggest that transcriptional activity is relatively preserved even at this stage. Altogether, it is unlikely that neuronal loss was induced by apoptosis. Necrosis, characterized by a gain in cell volume, swelling of organelles, plasma membrane rupture and subsequent loss of intracellular contents, ${ }^{38}$ is also unlikely as there were no findings to support it on morphology. On the other hand, autophagic cell death is morphologically defined as the absence of chromatin condensation with massive autophagic vacuolization of the cytoplasm. ${ }^{40}$ The electron microscopic analyses in this study showed that there were vacuoles in the 

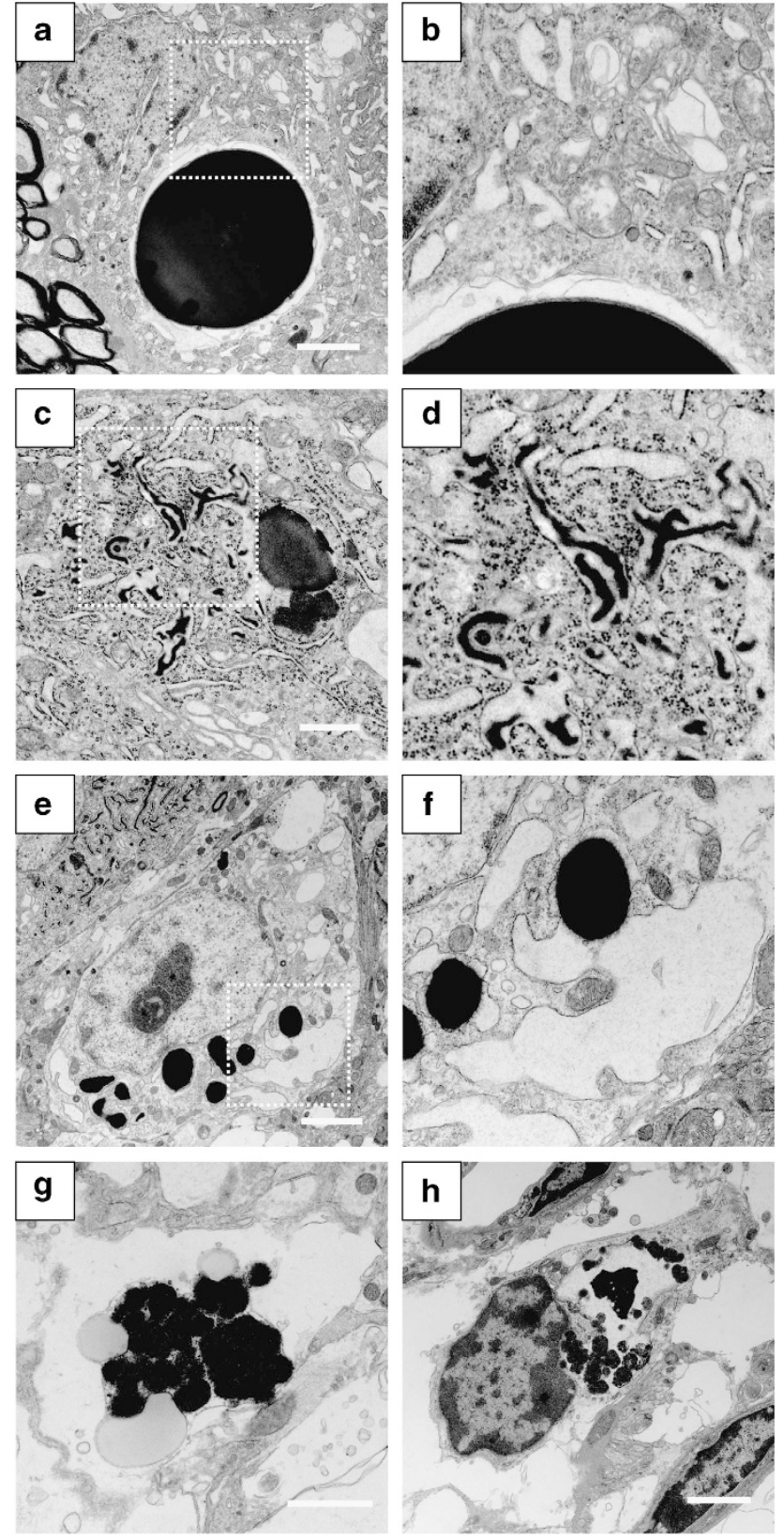

Figure 7 Electron microscopic analysis in AVP neurons of 5-month-old FNDI mice. AVP neurons of the SON in 5-month-old FNDI mice with water access ad libitum (a-d) and of those subjected to WD for 12 weeks (e-h). Higher magnification images of boxed areas in (a, $\mathbf{c}$ and $\mathbf{e})$ are shown in (b, $\mathbf{d}$ and $\mathbf{f})$, respectively. Scale bars, $2 \mu \mathrm{m}(\mathbf{a}, \mathbf{e}, \mathbf{g}$ and $\mathbf{h}), 1 \mu \mathrm{m}$ (c)

cytoplasm with aggregates inside. As our data also showed that autophagy was induced in AVP neurons in FNDI mice at least 4 weeks after starting WD, it is suggested that the cell death is associated with autophagy.

Although many studies showed that autophagy could cause cell death in non-mammalian organisms and mammalian cell lines, ${ }^{41}$ it has not been clear whether this type of cell death is related to the pathogenesis of any disease in humans. ERphagy as well as selective autophagy shown in this study should primarily be a protective and adaptive mechanism by
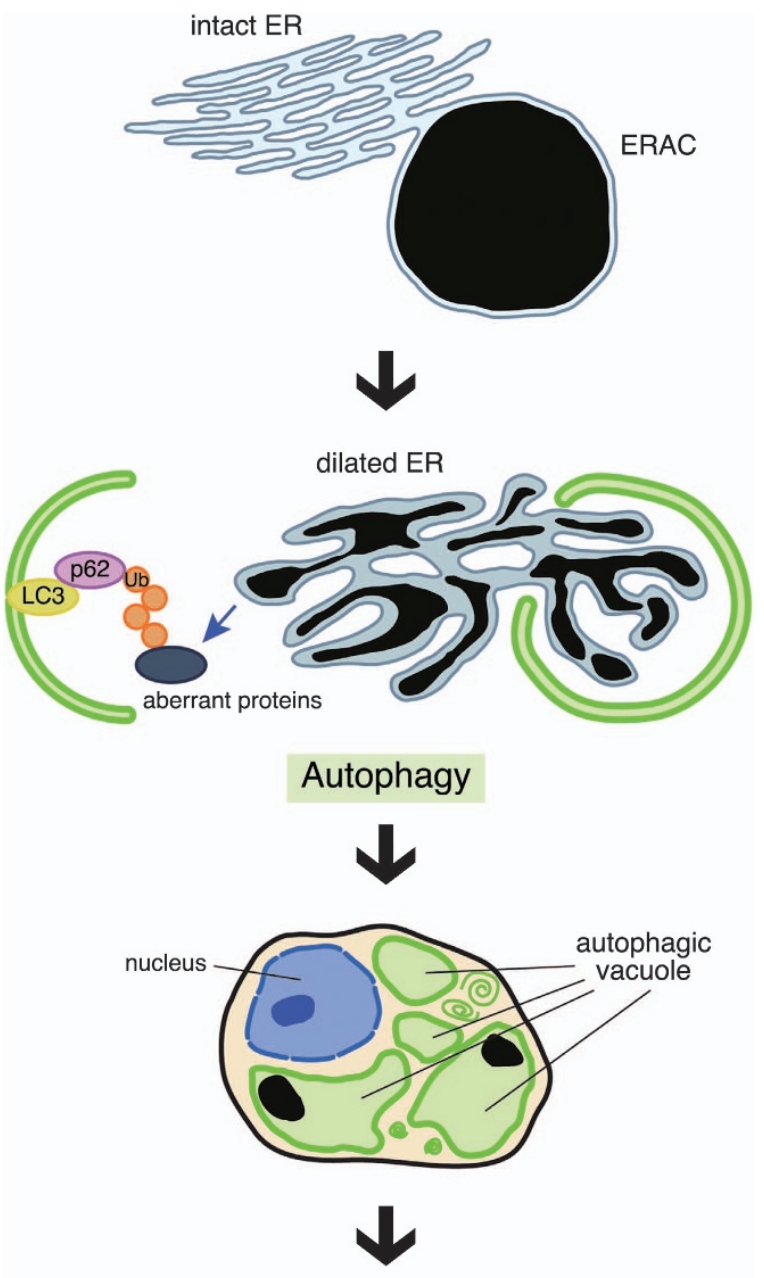

\section{Autophagy-associated cell death}

Figure 8 Possible mechanisms of cell death of AVP neurons in FNDI mice. Aggregates are confined to a sub-compartment of the ER, so-called ERAC, in AVP neurons of the SON in FNDI mice. Failure of ERAC formation leads to autophagy. When autophagy continues, the organelles including ER are lost over time, leading to autophagy-associated cell death

which misfolded proteins are degraded in the AVP neurons in FNDI mice. However, our data suggest that, if such autophagy is induced excessively and continues over time in order to cope with misfolded proteins, substantial amount of ER could be lost. Under these conditions, it would be difficult to maintain the ER homeostasis, and organelles other than ER would also be damaged and degraded through autophagy. Indeed, our data showed that the cytoplasm of some AVP neurons is almost entirely occupied with vacuoles in FNDI mice of 5 months old, when $30-40 \%$ AVP neurons are lost. We interpreted these data that AVP neurons are lost through 'autophagy-associated cell death', ${ }^{40}$ a term that is more appropriate than 'autophagic cell death' because of the original role of autophagy in the AVP neurons. Figure 8 illustrates the possible process and mechanisms of cell death of AVP neurons in FNDI mice.

The Brattleboro rat, another animal model for inherited neurohypophysial diabetes insipidus, carries a single- 
nucleotide deletion in the NPII-coding sequence of the AVP gene, which causes a frameshift mutation. ${ }^{42}$ The altered downstream region lacks the translational stop codon, and the mutant AVP precursor has a C-terminal polylysine stretch because of continued translation into the mRNA poly $(A)$ tail. In contrast to FNDI mice, the trait in the Brattleboro rats is recessive and only homozygous rats manifest the phenotype of polyuria, without apparent loss of AVP neurons. ${ }^{43}$ Although the mutant AVP precursor is not processed properly ${ }^{44,45}$ and reportedly retained within the $E R,{ }^{46}$ aggregates are barely detected in the ER of AVP neurons of Brattleboro rats. ${ }^{47,48}$ Thus, whether or not aggregates are formed in the ER may depend on conformational changes caused by mutant AVP precursors.

In conclusion, our data suggest that ERAC is formed in the AVP neurons of FNDI mice in order to maintain ER function or cell viability, and that failure of the compartmentation could lead to autophagy followed by autophagy-associated cell death in the AVP neurons in FNDI mice.

\section{Materials and Methods}

Animals. FNDI mice that were heterozygous for mutant gene (Cys98stop) were generated previously. ${ }^{7}$ GFP-LC3 transgenic mice (strain GFP-LC3\#53) carrying a rat LC3-enhanced GFP fusion construct under control of the chicken $\beta$-actin promoter with the cytomegalovirus immediate early enhancer ${ }^{49}$ were obtained from the RIKEN BioResource Center (Tsukuba, Japan). Ub ${ }^{\mathrm{G} 76 \mathrm{~V}}$-GFP transgenic mice (strain B6.Cg- $\mathrm{Tg}\left(\mathrm{CAG}-\mathrm{Ub}^{*} \mathrm{G} 76 \mathrm{~V} / \mathrm{GFP}\right) 2 \mathrm{Dant} / \mathrm{J}$ ) harboring a mutant ubiquitin moiety $\left(\mathrm{Ub}^{\mathrm{G} 76 \mathrm{~V}}\right)$-GFP fusion construct under control of the chicken $\beta$-actin promoter with the cytomegalovirus immediate early enhancer ${ }^{50}$ were purchased from the Jackson Laboratory (Bar Harbor, ME, USA). FNDI mice were mated with GFP-LC3 or Ub ${ }^{\mathrm{G} 76 \mathrm{~V}}$-GFP transgenic mice to generate FNDI/GFP-LC3 or FNDI/ $\mathrm{Ub}^{\mathrm{G} 76 \mathrm{~V}}$-GFP mice. C57BL/6J mice were purchased from Chubu Science Materials (Nagoya, Japan). Mice were maintained under controlled conditions $\left(23.0 \pm 0.5^{\circ} \mathrm{C}\right.$, lights on 0900 to 2100 hours), and male mice were used in the experiments except for hypothalamic organotypic cultures, in which both male and female pups were used. All procedures were approved by the Animal Experimentation Committee of the Nagoya University Graduate School of Medicine, and performed in accordance with the institutional guidelines for animal care and use.

Water deprivation. Two-month-old male mice were divided into ad libitum and WD groups. Mice in the ad libitum group had free access to water throughout the experiments, whereas those in the WD group were subjected to intermittent WD, which consists of repeated cycles of a continuous WD for $48 \mathrm{~h}$ followed by recovery period for 5 days (with free access to water). The intermittent WD was reported to accelerate the phenotype of FNDI model rats. ${ }^{25}$ Mice in the WD group were decapitated immediately after the last 48-h WD.

Measurements of urine volume and AVP. Mice were housed in metabolic cages, and the 24-h pooled urine was collected and measured on the fourth and fifth day of each recovery period (with free access to water). Urine AVP was measured using the Vasopressin RIA (Bühlmann Laboratories, Schönenbuch, Switzerland). The mean values of urine volumes and AVP were shown in each week.

Brain collection for immunohistochemistry and in situ hybridization. Mice were deeply anesthetized and transcardially perfused with a cold fixative containing $4 \%$ paraformaldehyde (PFA) in PBS, $\mathrm{pH}$ 7.4. After fixation, brains were removed and immersed in the same fixative for $2 \mathrm{~h}$ at $4{ }^{\circ} \mathrm{C}$. The brains were kept in PBS containing $10-20 \%$ sucrose at $4{ }^{\circ} \mathrm{C}$ for cryoprotection. They were embedded in Tissue-Tek O.C.T. compound (Sakura Finetechnical, Tokyo, Japan) and stored at $-80^{\circ} \mathrm{C}$ until sectioning. Brains were cut into $16 \mu \mathrm{m}$ sections on a cryostat at $-20^{\circ} \mathrm{C}$, thaw mounted on Superfrost Plus microscope slides (Matsunami, Tokyo, Japan), and stored at $-80^{\circ} \mathrm{C}$ until either immunohistochemistry or in situ hybridization.
Immunohistochemistry. The frozen sections were washed with PBS and $0.3 \%$ Triton X-100 in PBS, followed by blocking with a mixture of $5 \%$ normal goat serum and $3 \%$ bovine serum albumin in PBS for $1 \mathrm{~h}$ at room temperature (RT). For immunohistochemical studies, the sections were incubated with rabbit anti-mutant NPII (Cys98stop) antibody, which was generated previously overnight at $4{ }^{\circ} \mathrm{C}$. Primary antibody was probed using biotinylated goat anti-rabbit $\lg G(\mathrm{H}+\mathrm{L})$ (1:200; BA-1000; Vector Laboratories, Burlingame, CA, USA) for $3 \mathrm{~h}$ at RT. After being washed, the sections were incubated with avidin-biotin complex solution (1:100; Vectastatin ABC kit; Vector Laboratories) for $90 \mathrm{~min}$ at RT, and then immersed in PBS containing 0.1\% 3,3'-diaminobenzidine dihydrochloride (SigmaAldrich, St. Louis, MO, USA). Antibody-binding sites were made visible by adding $0.004 \%$ hydrogen peroxide. For immunofluorescence staining, sections were incubated with rabbit anti-mutant NPII (Cys98stop) antibody (1:1000), mouse antinormal NPII antibody (1: 100; kindly provided by Dr. H Gainer, National Institutes of Health, Bethesda, MD, USA), rabbit anti-p62 antibody (1:1000; PM045; Medical and Biological Laboratories, Nagoya, Japan) and mouse anti-GFP antibody (1:1000; A11120; Invitrogen, San Diego, CA, USA) overnight at $4{ }^{\circ} \mathrm{C}$. The sections were then treated with Alexa Fluor 488-conjugated goat anti-mouse IgG $(H+L)$ highly cross-adsorbed (1: 1000; A-11029; Invitrogen) and Alexa Fluor 568-conjugated goat anti-rabbit IgG $(\mathrm{H}+\mathrm{L})$ highly cross-adsorbed (1:1000; A-11036; Invitrogen) for $1 \mathrm{~h}$ at RT. Fluorescence images were acquired with a laser-scanning confocal microscope (LSM 5 Pascal; Carl Zeiss, Oberkochen, Germany) or a fluorescence microscope (BZ-9000; Keyence, Osaka, Japan) and processed using Adobe Photoshop CS5 (Adobe Systems, San Jose, CA, USA). TUNEL assay was performed using Apoptosis in situ Detection Kit (Wako Pure Chemical Industries Ltd, Osaka, Japan) according to the manufacturer's instructions.

In situ hybridization and quantification. The AVP intronic probe for hnRNA was constructed from a 685-bp fragment containing bases 171-855 of the mouse AVP intron 1 as described previously. ${ }^{8}$ The BiP exonic probe was created from a 922-bp fragment containing bases $852-1773$ of the mouse BiP cDNA as previously reported. ${ }^{51}$ Highly specific antisense probes of AVP hnRNA and BiP mRNA were synthesized using $55 \mu \mathrm{Ci} \quad{ }^{35}$ S JUTP and $\left.171 \mu \mathrm{Ci} \quad{ }^{35} \mathrm{~S}\right] \mathrm{CTP}$ (PerkinElmer Life Sciences, Natick, MA, USA), the Riboprobe Combination System (Promega, Madison, WI, USA), 15 units of RNasin, $1 \mu \mathrm{g}$ of linearized template and 15 units of SP6 RNA polymerase. After $60 \mathrm{~min}$ of incubation at $42^{\circ} \mathrm{C}$, the cDNA templates were digested with DNase for $10 \mathrm{~min}$ at $37^{\circ} \mathrm{C}$. Radiolabeled RNA products were purified using Quick Spin Columns for radiolabeled RNA purification (Roche Diagnostics, Indianapolis, IN, USA), precipitated with ethanol, and resuspended in $100 \mu$ of $10 \mathrm{mM}$ Tris- $\mathrm{HCl}, \mathrm{pH}$ 7.5, containing $20 \mathrm{mM}$ dithiothreitol. Prehybridization, hybridization and posthybridization procedures were performed as described previously. ${ }^{51}$ The sections were exposed to Kodak BioMax MR film (Kodak, Rochester, NY, USA) for various periods yielding appropriate signal intensities. The expression level of BiP mRNA in the SON was quantified by measurements of the integrated optimal densities (optic densities $\times$ area) of the film images using ImageJ software (version $1.44 \mathrm{k}$; http://rsbweb.nih.gov/ij/). The sections hybridized with AVP hnRNA probe were dipped in Kodak Autoradiography Emulsion Type NTB (Kodak) and exposed for 3 weeks. To assist cellular localization of the hybridized signals, the emulsion-dipped sections were counterstained with cresyl violet.

Electron microscopy. Mice were deeply anesthetized and then perfused with 4\% PFA in PBS. Brains were dissected and cut into $100 \mu \mathrm{m}$ sections on a vibratome (VT1200 S; Leica, Wetzlar, Germany). The sections were fixed in a mixture of $2 \%$ PFA and $2.5 \%$ glutaraldehyde in PBS overnight at $4{ }^{\circ} \mathrm{C}$, followed by postfixation with $2 \%$ osmium tetroxide for $20 \mathrm{~min}$ at $4{ }^{\circ} \mathrm{C}$. Each section was dehydrated in a graded ethanol series, treated with propylene oxide and embedded in epoxy resin (TAAB 812 Resin; TAAB Laboratories, Aldermaston, UK). Resin was polymerized for $48 \mathrm{~h}$ at $60^{\circ} \mathrm{C}$. After ultrathin sections $(70 \mathrm{~nm}$ thickness) including the SON were prepared with a diamond knife on an ultramicrotome (Reichert Ultracut S; Leica), they were counterstained with uranyl acetate and lead citrate, and analyzed with an electron microscope (JEM-1400EX; JEOL, Tokyo, Japan).

Counting AVP neurons in the SON. The cells expressing mutant NPII in immunohistochemistry and those expressing AVP hnRNA in in situ hybridization were counted in the SON of FNDI mice. The best-matched slice at $-0.7 \mathrm{~mm}$ 
caudal from the bregma, according to the brain atlas, ${ }^{52}$ was chosen in each mouse for the analysis. In in situ hybridization studies, any sections with grains of more than threefold the background density were considered labeled.

Measurement of number and diameter of inclusion bodies. For preparing semi-ultrathin sections ( $1 \mu \mathrm{m}$ thickness), epoxy resin-embedded samples including the SON were cut with a diamond knife on an ultramicrotome. The sections were mounted on glass slides and stained with toluidine blue. The number and diameter of the inclusion bodies in the SON were measured using an Olympus DP73 digital camera system and cellSens Software equipped with an Olympus BX51 microscope (Olympus, Tokyo, Japan).

Hypothalamic organotypic culture. Seven-day-old C57BL/6J, GFP-LC3 transgenic or Ub ${ }^{\mathrm{G} 76 \mathrm{~V}}$-GFP transgenic mice were decapitated and hypothalamic tissues ware sectioned at $350 \mu \mathrm{m}$ thickness on a Mcllwain tissue chopper (Mickle Laboratory Engineering Co., Surrey, UK). Three coronal slices containing the SON were separated and placed in Hank's balanced salt solution (Invitrogen) enriched with glucose. The selected sections were trimmed dorsally above the top of the third ventricle and laterally from the SON. Explants from each mouse were placed on Millicell-CM filter inserts (pore size, $0.4 \mu \mathrm{m}$; diameter, $30 \mathrm{~mm}$; Millipore, Billerica, MA, USA), and each filter insert was placed in a $35-\mathrm{mm}$ Petri dish. Culture medium was composed of 50\% MEM (Invitrogen), 25\% heat-inactivated horse serum (Invitrogen), $25 \%$ Hank's balanced salt solution, $20 \mathrm{units} / \mathrm{ml}$ penicillin and $20 \mu \mathrm{g} / \mathrm{ml}$ streptomycin (penicillin-streptomycin, liquid; Invitrogen), $1 \mathrm{mM} \mathrm{L-glutamine} \mathrm{(Invitrogen)} \mathrm{and} 0.5 \%$ glucose. Cultures were performed at $37^{\circ} \mathrm{C}$ in $5 \% \mathrm{CO}_{2}$ enriched air under stationary conditions. Explants were incubated with $1 \mu \mathrm{M}$ thapsigargin (Calbiochem, San Diego, CA, USA) or $1 \mu \mathrm{M}$ MG-132 (Calbiochem) for indicated time periods, while control slices were incubated with vehicle ( $0.1 \%$ dimethylsulfoxide; Sigma-Aldrich). Total duration of cultures was $24 \mathrm{~h}$ in all explants. For the evaluation of GFP signals in cultured hypothalamic slice explants of GFP-LC3 or Ub ${ }^{\mathrm{G} 76 \mathrm{~V}}$-GFP transgenic mice, the immunofluorescence staining for GFP was performed as described in the immunohistochemistry section, following wash with PBS and fixation in 4\% PFA. Fluorescence images were acquired with a laser-scanning confocal microscope (LSM 5 Pascal) or a fluorescence microscope (BZ-9000).

Immunoblotting. Mouse hypothalamic slice explants were lysed in a buffer

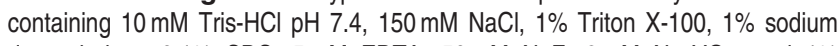
deoxycholate, $0.1 \%$ SDS, $5 \mathrm{mM}$ EDTA, $50 \mathrm{mM} \mathrm{NaF}, 2 \mathrm{mM} \mathrm{Na}_{3} \mathrm{VO}_{4}$, and $1 \%$ protease inhibitor cocktail (Sigma-Aldrich). After centrifuging the samples, protein concentrations in the supernatant were determined by bicinchoninic acid assay using a bicinchoninic acid kit (Sigma-Aldrich). Ten micrograms protein per sample was separated by $10 \%$ SDS-PAGE and transferred to polyvinylidene difluoride membranes (Millipore). Blots were blocked in $5 \%$ skimmed milk in TBS-T solution (10 $\mathrm{mM}$ Tris- $\mathrm{HCl} \mathrm{pH} 7.4,150 \mathrm{mM} \mathrm{NaCl}$ and $0.1 \%$ Tween) for $1 \mathrm{~h}$ at RT. Membranes were incubated with rabbit anti-p62 antibody (1:20000; PM045; Medical and Biological Laboratories) overnight at $4{ }^{\circ} \mathrm{C}$ and rabbit anti- $\beta$-actin antibody (1:20000; ab8227; Abcam, Cambridge, UK) for $1 \mathrm{~h}$ at RT. Primary antibodies were probed with HRP-conjugated donkey anti-rabbit IgG $(1: 20000$; NA934; GE Healthcare, Little Chalfont, UK) for $1 \mathrm{~h}$ at RT. To improve sensitivity and signal-to-noise ratio, Can Get Signal Immunoreaction Enhancer Solution (Toyobo, Osaka, Japan) was used for the dilution of the primary and secondary antibodies. Immunoreactivity was detected using the ECL Prime Western Blotting Detection Reagent (GE Healthcare).

Quantitative real-time RT-PCR. Total RNA was extracted from mouse hypothalamus slice explants using the RNeasy Mini kit (Qiagen, Chatsworth, CA, USA) according to the manufacturer's instructions. One microgram of total RNA was reverse-transcribed using ReverTra Ace qPCR RT Master Mix (Toyobo). Quantitative real-time RT-PCR reactions were carried out with Brilliant III UltraFast SYBR Green QPCR Master Mixes (Agilent Technologies, Palo Alto, CA, USA). The reaction condition was $95^{\circ} \mathrm{C}$ for $3 \mathrm{~min}$ and then 40 cycles of $20 \mathrm{~s}$ at $95^{\circ} \mathrm{C}$ and $20 \mathrm{~s}$ at $60^{\circ} \mathrm{C}$. As internal standard control, the expression of $18 \mathrm{~S}$ rRNA was simultaneously quantified. The primer sequences used were as follows: p62 mRNA, 5'-AGGATGGGGACTTGGTTGC-3' (forward), 5'-TCACAGATCACATTGG GGTGC-3' (reverse) and 18S rRNA, 5'-TTGACGGAAGGGCACCACCAG-3' (forward), 5'-GCACCACCACCCACGGAATCG-3' (reverse). Relative mRNA expression was calculated using the comparative $\mathrm{Ct}$ method and analyses were performed with Mx3000p qPCR system (Stratagene, La Jolla, CA, USA).
Statistical analysis. Statistical significance of the differences between groups was analyzed by either unpaired $t$-test, one-way ANOVA or two-way ANOVA with repeated measures followed by Bonferroni's test as appropriate. Results are expressed as means \pm S.E. and differences were considered statistically significant at $P<0.05$.

\section{Conflict of Interest}

The authors declare no conflict of interest.

Acknowledgements. This work was supported in part by a Grant-in-Aid for Scientific Research from the Japanese Society for Promotion of Science, Japan (23659475) and a Grant-in-Aid for Scientific Research (Research on Hypothalamohypophyseal Disorders) from the Ministry of Health, Labor and Welfare, Japan. We thank Dr. Noboru Mizushima (Tokyo University) for providing the GFP-LC3 transgenic mice. We are grateful to Michiko Yamada, Mayumi Katagiri and Kaori Ushida for their helpful technical assistance.

1. Robertson GL. Posterior pituitary. In: Felig P, Baxter JD, Frohman LA (eds) Endocrinology and Metabolism. McGraw-Hill: New York, 1995. pp 385-432.

2. Christensen JH, Rittig S. Familial neurohypophyseal diabetes insipidus-an update. Semin Nephrol 2006; 26: 209-223.

3. Babey M, Kopp P, Robertson GL. Familial forms of diabetes insipidus: clinical and molecular characteristics. Nat Rev Endocrinol 2011; 7: 701-714.

4. Ito M, Jameson JL. Molecular basis of autosomal dominant neurohypophyseal diabetes insipidus. Cellular toxicity caused by the accumulation of mutant vasopressin precursors within the endoplasmic reticulum. J Clin Invest 1997; 99: 1897-1905.

5. Birk J, Friberg MA, Prescianotto-Baschong C, Spiess M, Rutishauser J. Dominant pro-vasopressin mutants that cause diabetes insipidus form disulfide-linked fibrillar aggregates in the endoplasmic reticulum. J Cell Sci 2009; 122: 3994-4002.

6. Arima $\mathrm{H}$, Oiso $\mathrm{Y}$. Mechanisms underlying progressive polyuria in familial neurohypophysial diabetes insipidus. J Neuroendocrinol 2010; 22: 754-757.

7. Hayashi M, Arima H, Ozaki N, Morishita Y, Hiroi M, Nagasaki H et al. Progressive polyuria without vasopressin neuron loss in a mouse model for familial neurohypophysial diabetes insipidus. Am J Physiol Regul Integr Comp Physiol 2009; 296: R1641-R1649.

8. Morishita Y, Arima H, Hiroi M, Hayashi M, Hagiwara D, Asai N et al. Poly(A) tail length of neurohypophysial hormones is shortened under endoplasmic reticulum stress. Endocrinology 2011; 152: 4846-4855.

9. Blotner H. Primary or idiopathic diabetes insipidus: a system disease. Metabolism 1958; 7: 191-200.

10. Braverman LE, Mancini JP, McGoldrick DM. Hereditary idiopathic diabetes insipidus. A case report with autopsy findings. Ann Intern Med 1965; 63: 503-508.

11. Bergeron C, Kovacs K, Ezrin C, Mizzen C. Hereditary diabetes insipidus: an immunohistochemical study of the hypothalamus and pituitary gland. Acta Neuropathol 1991; 81: 345-348.

12. Ozcan L, Tabas I. Role of endoplasmic reticulum stress in metabolic disease and other disorders. Annu Rev Med 2012; 63: 317-328.

13. Carrell RW, Lomas DA. Alpha1-antitrypsin deficiency-a model for conformational diseases. N Engl J Med 2002; 346: 45-53.

14. Hagen MC, Murrell JR, Delisle MB, Andermann E, Andermann F, Guiot MC et al. Encephalopathy with neuroserpin inclusion bodies presenting as progressive myoclonus epilepsy and associated with a novel mutation in the proteinase inhibitor 12 gene. Brain Pathol 2011; 21: 575-582.

15. Støy J, Steiner DF, Park SY, Ye H, Philipson LH, Bell Gl. Clinical and molecular genetics of neonatal diabetes due to mutations in the insulin gene. Rev Endocr Metab Disord 2010; 11: 205-215.

16. Kraft $C$, Peter M, Hofmann K. Selective autophagy: ubiquitin-mediated recognition and beyond. Nat Cell Biol 2010; 12: 836-841.

17. Huyer G, Longsworth GL, Mason DL, Mallampalli MP, McCaffery JM, Wright RL et al. A striking quality control subcompartment in Saccharomyces cerevisiae: the endoplasmic reticulum-associated compartment. Mol Biol Cell 2004; 15: 908-921.

18. Valetti C, Grossi CE, Milstein C, Sitia R. Russell bodies: a general response of secretory cells to synthesis of a mutant immunoglobulin which can neither exit from, nor be degraded in, the endoplasmic reticulum. J Cell Biol 1991; 115: 983-994.

19. Kamhi-Nesher S, Shenkman M, Tolchinsky S, Fromm SV, Ehrlich R, Lederkremer GZ. A novel quality control compartment derived from the endoplasmic reticulum. Mol Biol Cell 2001; 12: 1711-1723.

20. Granell S, Baldini G, Mohammad S, Nicolin V, Narducci P, Storrie B. Sequestration of mutated alpha1-antitrypsin into inclusion bodies is a cell-protective mechanism to maintain endoplasmic reticulum function. Mol Biol Cell 2008; 19: 572-586.

21. Ito D, Yagi T, Ikawa M, Suzuki N. Characterization of inclusion bodies with cytoprotective properties formed by seipinopathy-linked mutant seipin. Hum Mol Genet 2012; 21: $635-646$. 
22. Castino R, Davies J, Beaucourt S, Isidoro C, Murphy D. Autophagy is a prosurviva mechanism in cells expressing an autosomal dominant familial neurohypophyseal diabetes insipidus mutant vasopressin transgene. FASEB J 2005; 19: 1021-1023.

23. Castino R, Isidoro C, Murphy D. Autophagy-dependent cell survival and cell death in an autosomal dominant familial neurohypophyseal diabetes insipidus in vitro model. FASEB 2005; 19: 1024-1026.

24. Castino R, Thepparit C, Bellio N, Murphy D, Isidoro C. Akt induces apoptosis in neuroblastoma cells expressing a C98X vasopressin mutant following autophagy suppression. J Neuroendocrinol 2008; 20: 1165-1175.

25. Si-Hoe SL, De Bree FM, Nijenhuis M, Davies JE, Howell LM, Tinley H et al. Endoplasmic reticulum derangement in hypothalamic neurons of rats expressing a familia neurohypophyseal diabetes insipidus mutant vasopressin transgene. FASEB $J 2000$ 14: $1680-1684$

26. Davies J, Murphy D. Autophagy in hypothalamic neurones of rats expressing a familial neurohypophysial diabetes insipidus transgene. J Neuroendocrinol 2002; 14 629-637.

27. Yorimitsu T, Nair U, Yang Z, Klionsky DJ. Endoplasmic reticulum stress triggers autophagy J Biol Chem 2006; 281: 30299-30304.

28. Ogata M, Hino S, Saito A, Morikawa K, Kondo S, Kanemoto S et al. Autophagy is activated for cell survival after endoplasmic reticulum stress. Mol Cell Biol 2006; 26 : 9220-9231

29. Bernales $\mathrm{S}, \mathrm{McD}$ onald $\mathrm{KL}$, Walter $\mathrm{P}$. Autophagy counterbalances endoplasmic reticulum expansion during the unfolded protein response. PLoS Biol 2006; 4: e423.

30. Teckman JH, Perlmutter DH. Retention of mutant alpha(1)-antitrypsin Z in endoplasmic reticulum is associated with an autophagic response. Am J Physiol Gastrointest Liver Physiol 2000; 279: G961-G974.

31. Kamimoto T, Shoji S, Hidvegi T, Mizushima N, Umebayashi K, Perlmutter DH et al. Intracellular inclusions containing mutant alpha1-antitrypsin $Z$ are propagated in the absence of autophagic activity. J Biol Chem 2006; 281: 4467-4476.

32. Smith MH, Ploegh HL, Weissman JS. Road to ruin: targeting proteins for degradation in the endoplasmic reticulum. Science 2011; 334: 1086-1090.

33. Shaid S, Brandts $\mathrm{CH}$, Serve H, Dikic I. Ubiquitination and selective autophagy. Cell Death Differ 2013; 20: 21-30.

34. Kuusisto $E$, Suuronen $T$, Salminen A. Ubiquitin-binding protein $p 62$ expression is induced during apoptosis and proteasomal inhibition in neuronal cells. Biochem Biophys Res Commun 2001; 280: 223-228.

35. Nakaso K, Yoshimoto $\mathrm{Y}$, Nakano T, Takeshima T, Fukuhara $\mathrm{Y}$, Yasui $\mathrm{K}$ et al. Transcriptional activation of p62/A170/ZIP during the formation of the aggregates: possible mechanisms and the role in Lewy body formation in Parkinson's disease. Brain Res 2004; 1012: 42-51.

36. Klionsky DJ, Abdalla FC, Abeliovich H, Abraham RT, Acevedo-Arozena A, Adeli K et al. Guidelines for the use and interpretation of assays for monitoring autophagy. Autophagy 2012; 8: 445-544.

37. Liu XD, Ko S, Xu Y, Fattah EA, Xiang Q, Jagannath $C$ et al. Transient aggregation of ubiquitinated proteins is a cytosolic unfolded protein response to inflammation and endoplasmic reticulum stress. J Biol Chem 2012; 287: 19687-19698.

38. Kroemer G, Galluzzi L, Vandenabeele P, Abrams J, Alnemri ES, Baehrecke EH et al. Classification of cell death: recommendations of the Nomenclature Committee on Cell Death 2009. Cell Death Differ 2009 16: 3-11.
39. Hotchkiss RS, Strasser A, McDunn JE, Swanson PE. Cell death. N Engl J Med 2009; 361: $1570-1583$.

40. Kroemer G, Levine B. Autophagic cell death: the story of a misnomer. Nat Rev Mol Cell Biol 2008; 9: 1004-1010.

41. Gozuacik D, Kimchi A. Autophagy and cell death. Curr Top Dev Biol 2007; 78: 217-245.

42. Schmale H, Richter D. Single base deletion in the vasopressin gene is the cause of diabetes insipidus in Brattleboro rats. Nature 1984; 308: 705-709.

43. Szot P, Dorsa DM. Cytoplasmic and nuclear vasopressin RNA in hypothalamic and extrahypothalamic neurons of the Brattleboro rat: an in situ hybridization study. Mol Cell Neurosci 1992; 3: 224-236.

44. Kim JK, Summer SN, Wood WM, Brown JL, Schrier RW. Arginine vasopressin secretion with mutants of wild-type and Brattleboro rats AVP gene. J Am Soc Nephrol 1997; 8: 1863-1869.

45. Iwasaki Y, Oiso Y, Saito H, Majzoub JA. Effects of various mutations in the neurophysin/ glycopeptide portion of the vasopressin gene on vasopressin expression in vitro. Tohoku J Exp Med 2000; 191: 187-202.

46. Krisch B, Nahke P, Richter D. Immunocytochemical staining of supraoptic neurons from homozygous Brattleboro rats by use of antibodies against two domains of the mutated vasopressin precursor. Cell Tissue Res 1986; 244: 351-358.

47. Kalimo H, Rinne UK. Ultrastructural studies on the hypothalamic neurosecretory neurons of the rat. II. The hypothalamo-neurohypophysial system in rats with hereditary hypothalamic diabetes insipidus. Z Zellforsch Mikrosk Anat 1972; 134: 205-225.

48. Tasso F, Rua S. Ultrastructural observations on the hypothalamo-posthypophysical complex of the Brattleboro rat. Cell Tissue Res 1978; 191: 267-286.

49. Mizushima N, Yamamoto A, Matsui M, Yoshimori T, Ohsumi Y. In vivo analysis of autophagy in response to nutrient starvation using transgenic mice expressing a fluorescent autophagosome marker. Mol Biol Cell 2004; 15: 1101-1111.

50. Lindsten K, Menendez-Benito V, Masucci MG, Dantuma NP. A transgenic mouse model of the ubiquitin/proteasome system. Nat Biotechnol 2003; 21: 897-902.

51. Hagiwara D, Arima H, Morishita Y, Goto M, Banno R, Sugimura Y et al. BiP mRNA expression is upregulated by dehydration in vasopressin neurons in the hypothalamus in mice. Peptides 2012; 33: 346-350.

52. Paxinos G, Franklin KJ. The Mouse Brain in Stereotaxic Coordinates. Academic Press: New York, 2000

(2) ()ㅇ Cell Death and Disease is an open-access journal BY No no published by Nature Publishing Group. This work is licensed under a Creative Commons Attribution-NonCommercialNoDerivs 3.0 Unported License. The images or other third party material in this article are included in the article's Creative Commons license, unless indicated otherwise in the credit line; if the material is not included under the Creative Commons license, users will need to obtain permission from the license holder to reproduce the material. To view a copy of this license, visit http://creativecommons.org/licenses/ by-nc-nd/3.0/

Supplementary Information accompanies this paper on Cell Death and Disease website (http://www.nature.com/cddis) 